\title{
Chemical Availability of Bromide Dictates CsPbBr3 Nanocrystal Growth
}

Je-Ruei Wen, Benjamin Roman, Freddy Rodriguez Ortiz, Noel Mireles Villegas, Nicholas Porcellino, Matthew Sheldon

Submitted date: 05/05/2019 - Posted date: 06/05/2019

Licence: CC BY-NC-ND 4.0

Citation information: Wen, Je-Ruei; Roman, Benjamin; Rodriguez Ortiz, Freddy; Mireles Villegas, Noel; Porcellino, Nicholas; Sheldon, Matthew (2019): Chemical Availability of Bromide Dictates CsPbBr3 Nanocrystal Growth. ChemRxiv. Preprint.

Lack of detailed understanding of the growth mechanism of $\mathrm{CsPbBr} 3$ nanocrystals has hindered sophisticated morphological and chemical control of this important emerging optoelectronic material. Here, we have elucidated the growth mechanism by slowing the reaction kinetics. When 1-bromohexane is used as an alternative halide source, bromide is slowly released into the reaction mixture, extending the reaction time from $\sim 3$ seconds to greater than 20 minutes. This enables us to monitor the phase evolution of products over the course of reaction, revealing that $\mathrm{CsBr}$ is the initial species formed, followed by $\mathrm{Cs} 4 \mathrm{PbBr}$, and finally $\mathrm{CsPbBr} 3$. Further, formation of monodisperse $\mathrm{CsBr}$ nanocrystals is demonstrated in a bromide-deficient and lead-abundant solution. The $\mathrm{CsBr}$ can only be transformed into $\mathrm{CsPbBr} 3$ nanocubes if additional bromide is added. Our results indicate a fundamentally different growth mechanism for $\mathrm{CsPbBr} 3$ in comparison with more established semiconductor nanocrystal systems and reveal the critical role of the chemical availability of bromide for the growth reactions.

File list (2)

Chemical Availability of Bromide Dictates CsPbBr3 Nanocr... (1.08 MiB) view on ChemRxiv • download file SI --Chemical Availability of Bromide Dictates CsPbBr3 Na... (1.04 MiB) view on ChemRxiv • download file 


\title{
Chemical Availability of Bromide Dictates CsPbBr 3 Nanocrystal Growth
}

Je-Ruei Wen, ${ }^{\dagger}$ Benjamin J. Roman, ${ }^{\dagger}$ Freddy A. Rodriguez Ortiz,${ }^{\dagger}$ Noel Mireles Villegas, ${ }^{\dagger}$ Nicholas Porcellino, ${ }^{\dagger}$ Matthew T. Sheldon* ${ }^{* \dagger}$

${ }^{\dagger}$ Department of Chemistry and Department of Material Science and Engineering, Texas A\&M University, College Station, Texas 77843, United States.

\begin{abstract}
Lack of detailed understanding of the growth mechanism of $\mathrm{CsPbBr}_{3}$ nanocrystals has hindered sophisticated morphological and chemical control of this important emerging optoelectronic material. Here, we have elucidated the growth mechanism by slowing the reaction kinetics. When 1-bromohexane is used as an alternative halide source, bromide is slowly released into the reaction mixture, extending the reaction time from $\sim 3$ seconds to greater than 20 minutes. This enables us to monitor the phase evolution of products over the course of reaction, revealing that $\mathrm{CsBr}$ is the initial species formed, followed by $\mathrm{Cs}_{4} \mathrm{PbBr}_{6}$, and finally $\mathrm{CsPbBr}_{3}$. Further, formation of monodisperse $\mathrm{CsBr}$ nanocrystals is demonstrated in a bromide-deficient and lead-abundant solution. The $\mathrm{CsBr}$ can only be transformed into $\mathrm{CsPbBr}_{3}$ nanocubes if additional bromide is added. Our results indicate a fundamentally different growth mechanism for $\mathrm{CsPbBr}_{3}$ in comparison with more established semiconductor nanocrystal systems and reveal the critical role of the chemical availability of bromide for the growth reactions.
\end{abstract}

\section{Main text}

Rapid injection of organometallic precursors into high temperature solutions of surfactant ligands has been long established as an optimal synthetic strategy for the preparation of colloidal semiconductor nanocrystals. ${ }^{1}$ With proper understanding of growth dynamics, nanomaterials with tailored sizes, morphologies, chemical compositions, and surface chemistry can be prepared. ${ }^{2}$ This synthetic route 
currently produces the highest quality cesium lead halide perovskites $\left(\mathrm{CsPbX}_{3}, \mathrm{X}=\mathrm{Cl}, \mathrm{Br}, \mathrm{I}\right)$, a family of semiconductor nanocrystals that has recently drawn intense interest due to high photoluminescence quantum yield, ${ }^{3}$ highly tunable bandgap energy, ${ }^{4}$ ultrafast carrier dynamics,,${ }^{5,6}$ and efficient photon upand down-conversions, ${ }^{7,8,9,10}$ among many other ideal optoelectronic properties. ${ }^{11}$ Precisely defined nanocrystal dimensions from a few to tens of nanometers, ${ }^{12,13}$ as well as varied morphologies such as nanocubes, nanowires, ${ }^{14,15}$ and nanoplatelets/nanosheets, ${ }^{16,17,18}$ are now routinely synthesized. Successful doping of $\mathrm{CsPbX}_{3}$ nanocrystals has also been acheived, ${ }^{9,}{ }^{19}$ making it possible to further engineer the electrical and optical properties.

Despite this progress, fundamental understanding of the growth mechanism of perovskite nanocrystals is very limited. Owing to the strongly ionic nature of $\mathrm{CsPbX}_{3}$, nanoparticle growth finishes just a few seconds after injection, ${ }^{12,20}$ greatly hindering mechanistic studies. This challenge is further compounded by observations that synthetic strategies for structural control established in other semiconductor systems, such as seeded growth, ${ }^{21}$ variations in precursor concentration, ${ }^{22}$ or modified growth temperature, ${ }^{23,} 24$ rarely induce the anticipated result in a perovskite synthesis, and often alter the final product minimally, if at all. For these reasons more sophisticated control over morphology remains elusive. Better understanding of the $\mathrm{CsPDX}_{3}$ growth mechanism may enable highly desirable morphological targets, such as nanowires and nanosheets with controllable aspect ratios, ${ }^{25}$ or well-defined multi-component heterostructures, ${ }^{26,27}$ of which only limited examples have been demonstrated to date.

In the conventional $\mathrm{CsPbBr}_{3}$ synthetic protocol established by Protesescu et al, ${ }^{12}$ cesium oleate (Cs-OA) is injected into a solution of 1 -octadecene (ODE) containing $\mathrm{PbBr}_{2}$, oleic acid (OA) and oleylamine $(\mathrm{OAm}) . \mathrm{The}^{2+}$ and $\mathrm{Br}^{-}$precursors are present in the forms of lead oleate $(\mathrm{Pb}-\mathrm{OA})$ and oleylammonium 
bromide (OAm-Br) ${ }^{28}$ and the injection of Cs-OA leads to a reaction that completes within $\sim 3$ seconds. It has been proposed that the growth starts with $\left[\mathrm{PbBr}_{6}\right]^{4-}$ scaffolds, followed by the incorporation of $\mathrm{Cs}^{+}{ }^{29}$, ${ }^{30}$ In this proposal, the nanocrystal growth obeys a classical LaMer mechanism, in which the growth is initiated by the formation of single-species nuclei that subsequently grow into larger particles. ${ }^{31}$ Nevertheless, this mechanism cannot explain well the formation of some $\mathrm{CsPbX}_{3}$ morphologies, e.g. micrometers-long nanowires,${ }^{14}$ since none of the expected intermediate species have been observed.

In this work, we provide an alternative description of $\mathrm{CsPbBr}_{3}$ nanoparticle growth. Using 1-bromohexane as a halide precursor extends the time scale of growth from $\sim 3$ seconds to tens of minutes. This slow growth allows us to monitor, ex situ, the evolution of products in the early stages of $\mathrm{CsPbBr}_{3}$ nanoparticle formation. Using a combination of powder X-ray diffraction (XRD) and high-resolution transmission electron microscopy (HRTEM), we observe the initial formation of $\mathrm{CsBr}$, followed by $\mathrm{Cs}_{4} \mathrm{PbBr}_{6}$, and eventually $\mathrm{CsPbBr}_{3}$. Apart from the slow release of bromide, we show that the chemical environment during synthesis remains largely identical to that of a conventional preparation. Thus, our results suggest that the complexation of $\mathrm{Br}^{-}$to $\mathrm{Cs}^{+}$is, surprisingly, the critical step that initiates $\mathrm{CsPbr}_{3}$ nanoparticle nucleation, with $\mathrm{Cs}_{4} \mathrm{PbBr}_{6}$ playing the role of intermediate. Furthermore, we show that the crystal phase of products is determined by the concentration of bromide but independent of the concentration of lead. Even in a lead-abundant solution, pure monodisperse $\mathrm{CsBr}$ nanocrystals are produced by limiting the availability of bromide. The $\mathrm{CsBr}$ nanocrystals serve as seeds and undergo the evolution to monodisperse $\mathrm{CsPbr}_{3}$ nanocubes by addition of 1-bromohexane, but not by injecting extra $\mathrm{Pb}$-OA. These results unambiguously indicate that the chemical availability of bromide not only determines the kinetics, but also exclusively dictates the thermodynamic equilibrium of the growth products. 


\section{Mechanistic Study via Slow Release of Bromide}

For our alternative synthesis, $\mathrm{Pb}-\mathrm{OA}$ was individually prepared and mixed with Cs-OA in ODE. Following the addition of OA and OAm, 1-bromohexane was injected into the mixture. When 1-bromohexane is used as the halide source, bromide is gradually released from the precursor, limiting its chemical availability and extending the reaction time from a few seconds to tens of minutes. Aliquots were removed over the course of reaction for further analysis. More experimental details can be found in the Methods.

(a)

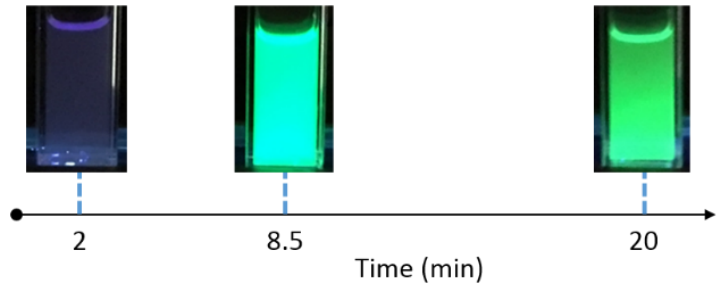

(b)

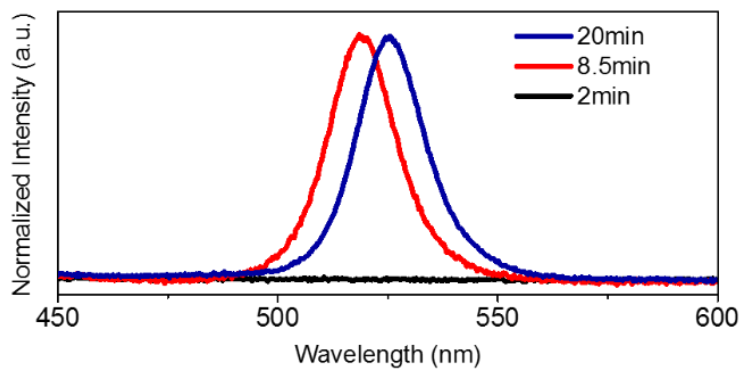

(c)

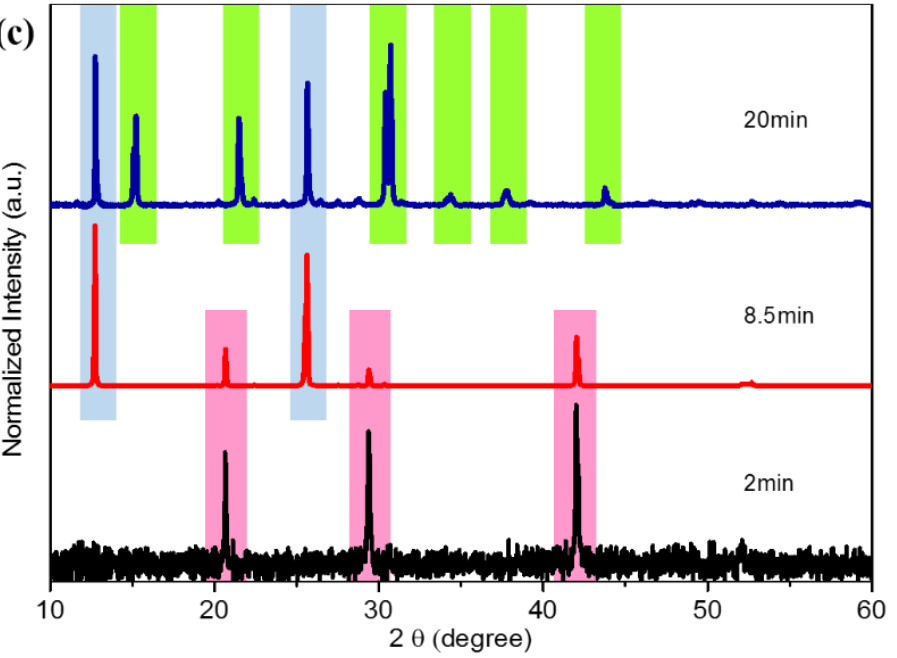

Figure 1. (a) Photos, (b) PL spectra, and (c) XRD patterns of samples collected at different reaction time. The photos were taken under UV light illumination. In (c), $\mathrm{CsBr}, \mathrm{Cs}_{4} \mathrm{PbBr}_{6}$ and $\mathrm{CsPbBr}_{3}$ peaks (COD ID 9008788, 1538416, 4510745) are labeled in pink, blue and green, respectively.

As shown in Figure 1a, the solution remained clear and non-fluorescent for $\sim 2$ minutes after 1bromohexane was injected. After 2 minutes, the solution started to turn turbid and white while remaining non-fluorescent (Figure 1b). After approximately 8.5 minutes, the mixture gradually became cloudier and showed photoluminescence (PL) with a peak centered at $517 \mathrm{~nm}$. After 20 minutes, the sample appeared 
fluorescent green, and the PL peak shifted to $525 \mathrm{~nm}$. No obvious change was observed beyond 20 minutes.

The crystal phase of the product at each stage was monitored ex-situ using XRD (Figure 1c). The white precipitate collected at 2 minutes exhibits the pattern of cubic $\mathrm{CsBr}$. The $\mathrm{CsBr}$ peaks decrease in intensity after 8.5 minutes, whereas the pattern of hexagonal $\mathrm{Cs}_{4} \mathrm{PbBr}_{6}$, which may be regarded as a lead halidedeficient phase of the perovskite, appears as the dominant feature, along with a weak $\mathrm{CsPbBr}_{3}$ perovskite signal. As the reaction proceeds to 20 minutes, the pattern shows clear $\mathrm{Cs}_{4} \mathrm{PbBr}_{6}$ and orthorhombic $\mathrm{CsPbBr}_{3}$ features, while the peaks from $\mathrm{CsBr}$ completely vanish. These transitions are further evident in the $\mathrm{PL}$ over time. $\mathrm{CsBr}$ and $\mathrm{Cs}_{4} \mathrm{PbBr}_{6}$ are insulators, explaining the lack of $\mathrm{PL}$ before 8.5 minutes. The trace amount of $\mathrm{CsPbBr}_{3}$ found at 8.5 minutes accounts for the cyan-green PL, whereas the red shift in PL peaks indicates the growth of luminescent $\mathrm{CsPbBr}_{3}$ nanocrystals over time.

This analysis was also confirmed by HRTEM. The image of the sample taken at 2 minutes (Figures S1a,b) depicts straw-like shapes, exhibiting a lattice fringe of $3.0 \AA$ that can be assigned to the $\left(\begin{array}{lll}1 & 1 & 0\end{array}\right)$ plane of cubic CsBr. At 8.5 minutes (Figures S1c,d), 200-300 nm-sized hexagons were observed. The lattice fringes shown in Figure S1d are 6.8 and $4.0 \AA$ A, corresponding to $\left(\begin{array}{lll}1 & 1 & 0\end{array}\right)$ and $\left(\begin{array}{lll}3 & 0 & 0\end{array}\right)$ planes of $\mathrm{Cs}_{4} \mathrm{PbBr}_{6}$, respectively. For the precipitate collected at 20 minutes (Figures S1e,f), the product is composed of 100$200 \mathrm{~nm}$-sized cuboidal structures, which is the common shape of $\mathrm{CsPbBr}_{3}$ nanocrystals. The lattice fringe identified in Figure $2 \mathrm{f}$ is $5.8 \AA$, which matches well with $(\overline{1} 0 \overline{1})$ plane of orthorhombic $\mathrm{CsPbBr}_{3}$.

As the reaction progressed beyond 8.5 minutes, more $\mathrm{CsPbBr}_{3}$ dominated the reaction. The presence of $\mathrm{Cs}_{4} \mathrm{PbBr}_{6}$ in the final product here may be attributed to the acid-base equilibrium of ligands, which is an important factor in determining the product phase. ${ }^{23}$ In fact, by increasing the $\mathrm{Br}^{-}$concentration while 
decreasing OAm (Table S1), pure perovskite crystals could be synthesized in this scheme (Figures S2 and S3). Yet, modifying the parameters in this manner also increased the reaction rate, leading to difficulty isolating the early products.

What makes tracking of the crystal evolution viable is the slow release of bromide from 1-bromohexane, likely through an $S_{N} 2$ reaction with oleylamine or an E2 reaction with oleate. Since free ions are insoluble in the nonpolar solution, the released bromide is expected to be present in the forms of OAm-Br, as with a conventional synthesis, or a secondary ammonium salt, which is the product of the $\mathrm{S}_{\mathrm{N}} 2$ reaction. This approach is distinct from other protocols utilizing alternative halide precursors, such as benzoyl bromide, ${ }^{32}$ trimethylsilyl bromide (TMSBr), ${ }^{30} \mathrm{OAm}-\mathrm{Br},{ }^{33}$ or trioctylphosphine- $\mathrm{Br}_{2}\left(\mathrm{TOP}-\mathrm{Br}_{2}\right) .{ }^{34}$ Protocols utilizing these precursors have reactions kinetics that complete in seconds, without a perceptible transition of products over time. It is the choice of halide source, not the separation of precursors, that is responsible for the slowed growth when using 1-bromohexane. Additionally, other reaction parameters such as the accessibility of cesium, precursor concentrations, alkylammonium and carboxylate ligand choices, or reaction temperature do not show similar effects on the growth rate. ${ }^{23,34,35,36}$ Seemingly, only the chemical availability of halide plays a crucial role in defining the reaction kinetics.

The slow $\mathrm{Br}^{-}$release was further confirmed in a separate experiment by allowing incubation time for 1bromohexane to generate sufficient bromide species before the final injection. To demonstrate this, a mixture of $\mathrm{Pb}-\mathrm{OA}, \mathrm{OA}, \mathrm{OAm}$, and 1-bromohexane was aged for $30 \mathrm{~min}$ at $150{ }^{\circ} \mathrm{C}$. Cs-OA was then injected, and $\mathrm{CsPBBr}_{3}$ nanocubes formed instantly (Figure S4, see SI for detail), as in the conventional synthesis. This incubation time study not only supports the proposed mechanism of slow bromide release, but also verifies that 1-bromohexane and any byproducts do not otherwise modify the nanoparticle growth. 
Thus, this control experiment also confirms that the growth steps observed during the slowed reaction may be viewed as representative of the growth dynamics in the general synthesis, albeit with slower rates of bromide incorporation.

The above results indicate the evolution of dominant products at each stage in the $\mathrm{Cs}-\mathrm{Pb}-\mathrm{Br}$ system, as summarized in equation (1):

$$
\mathrm{Cs}^{+}+\mathrm{Pb}^{2+} \stackrel{\mathrm{Br}^{-}}{\longrightarrow} \mathrm{CsBr} \stackrel{B r^{-}}{\longrightarrow} \mathrm{Cs}_{4} \mathrm{PbBr}_{6} \stackrel{\mathrm{Br}^{-}}{\longrightarrow} \mathrm{CsPbBr}_{3}
$$

As the reaction progresses, the dominant product changes from $\mathrm{CsBr}$ to $\mathrm{Cs}_{4} \mathrm{PbBr}_{6}$ to the final $\mathrm{CsPbBr}_{3}$. Since the bromide availability was essentially the only variable in our study, this evolution reflects that in the early stage of all hot injection syntheses of $\mathrm{CsPbr}_{3}, \mathrm{Br}^{-}$is expected to individually complex with $\mathrm{Cs}^{+}$ even with the presence of $\mathrm{Pb}^{2+}$. This mechanism does not preclude the formation of $\left[\mathrm{PbBr}_{6}\right]^{4-}$ octahedra in solution, however the ionic compound, $\mathrm{CsBr}$, is the first compound to crash out of solution, rather than a lead-containing species. As more bromide ions become available, $\mathrm{Cs}_{4} \mathrm{PbBr}_{6}$ and $\mathrm{CsPbr}_{3}$ phases become the prevailing products. The polydispersity of the product prepared using 1-bromohexane (Figure S1) is a direct consequence of this continual evolution of products. Bromide is slowly released from 1bromohexane throughout the reaction, leading to the continual nucleation and growth of $\mathrm{CsBr}$ simultaneous to the following phase conversions. Once all the Cs-OA has been converted to $\mathrm{CsBr}$, nucleation ceases and only phase conversion occurs. 

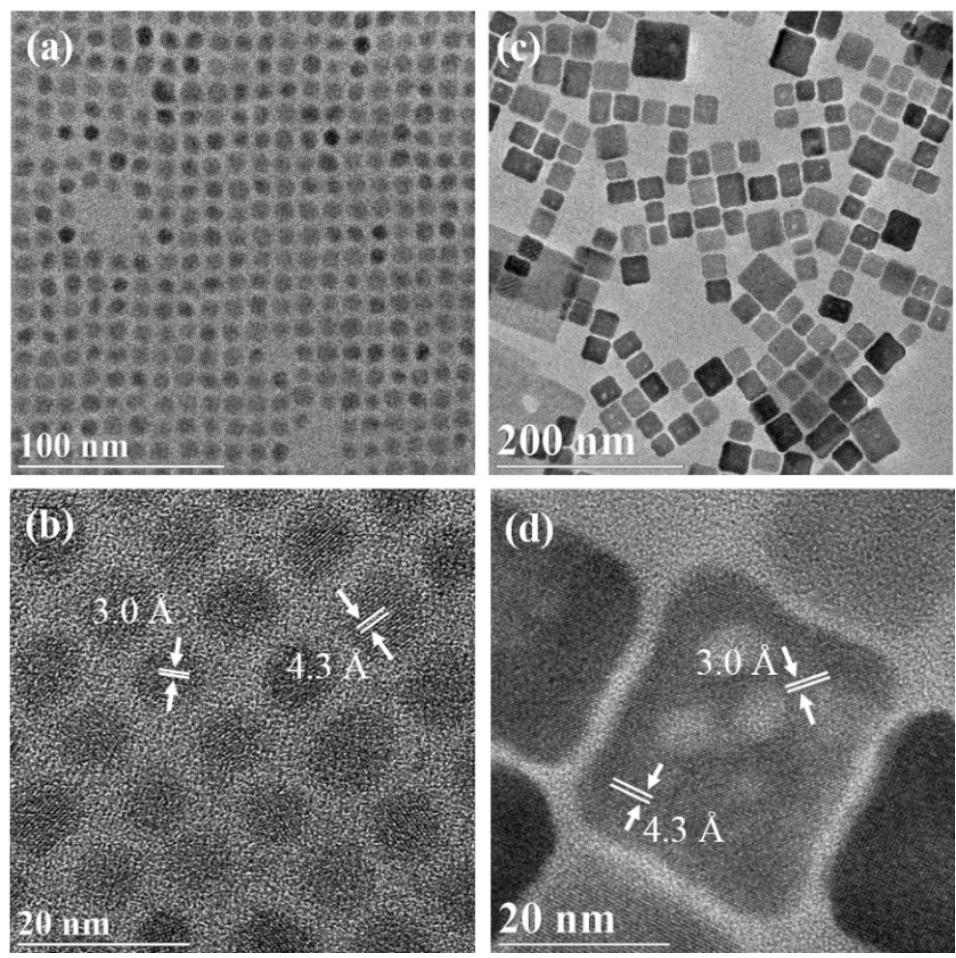

Figure 2. HRTEM images of $\mathrm{CsBr}$ nanocrystals prepared at $(\mathrm{a}, \mathrm{b}) 80^{\circ} \mathrm{C}$ and $(\mathrm{c}, \mathrm{d}) 150{ }^{\circ} \mathrm{C}$. The fringes measured to be $4.3 \AA$ and $3.0 \AA$ A correspond to ( $\left.\begin{array}{lll}1 & 0 & 0\end{array}\right)$ and $\left(\begin{array}{lll}1 & 1 & 0\end{array}\right)$ of CsBr, respectively.

\section{Slow Growth with Monodisperse CsBr Nanocrystal Seeds}

This new understanding of the role of bromide in the Cs- $\mathrm{Pb}-\mathrm{Br}$ reaction system extends beyond just the slow reaction kinetics of 1-bromohexane. Monodisperse CsBr nanocrystals can also be synthesized with $\mathrm{PbBr}_{2}$ in a hot injection reaction analogous to the conventional $\mathrm{CsPbBr}_{3}$ synthesis (Figures 2, 3a,c,d, S5, see Methods for detail). This is accomplished by reducing the ratio of $\mathrm{PbBr}_{2}$ to Cs-OA relative to what is standardly used to prepare $\mathrm{CsPBr}_{3}$, limiting the availability of bromide in order to prevent the evolution of a final product beyond the $\mathrm{CsBr}$ phase. Even when the lead concentration is increased with the addition of $\mathrm{Pb}-\mathrm{OA}$, the system still prefers the formation of $\mathrm{CsBr}$ nanocrystals (Figure $\mathrm{S6}$ ), despite having stoichiometrically sufficient $\mathrm{Pb}$ to produce $\mathrm{Cs}_{4} \mathrm{PbBr}_{6}$ or $\mathrm{CsPbBr}_{3}$. Such preference of phase of products is independent of the injection order of $\mathrm{Cs}$ and extra $\mathrm{Pb}$ precursors. In contrast, when 1-bromohexane is 
added to the $\mathrm{CsBr}$ crude solution, the product phase begins to evolve, as with the slow growth discussed previously. Pure, monodisperse $\mathrm{Cs}_{\mathrm{PbBr}}$ nanocrystals can be achieved with the slow kinetics dictated by the bromide release from 1-bromohexane when sufficient concentration of all precursors is present.

A representative example of this reaction is presented in Figure 3. Following the preparation of $\mathrm{CsBr}$ seeds, 1-bromohexane and $\mathrm{Pb}$-OA were injected into the crude solution. The solution turned weakly fluorescent 10 min after the injection, with a PL peak at $513 \mathrm{~nm}$ (Figure 3b). The PL peak gradually red-shifted to 516 and $520 \mathrm{~nm}$ around $26 \mathrm{~min}$ and 2 hours, respectively. The phase evolution was monitored ex situ by $\mathrm{XRD}$, as shown in Figure 3a. The products at 10 min showed a major pattern of $\mathrm{CsBr}$ along with minor $\mathrm{Cs}_{4} \mathrm{PbBr}_{6}$ signals. At around 16 min, the pattern of $\mathrm{Cs}_{5} \mathrm{PbBr}_{3}$ perovskite appeared. The patterns of $\mathrm{CsBr}$ and $\mathrm{Cs}_{4} \mathrm{PbBr}_{6}$ were greatly reduced at 26 min, whereas diffraction peaks assigned to orthorhombic $\mathrm{CsPbBr}_{3}$ became the dominant feature. At 2 hours, the pure orthorhombic perovskite phase was achieved. This was also confirmed by HRTEM, which showed $\mathrm{CsPbBr}_{3}$ nanocubes with sizes around 10-12 nm (Figures 3e,f). 

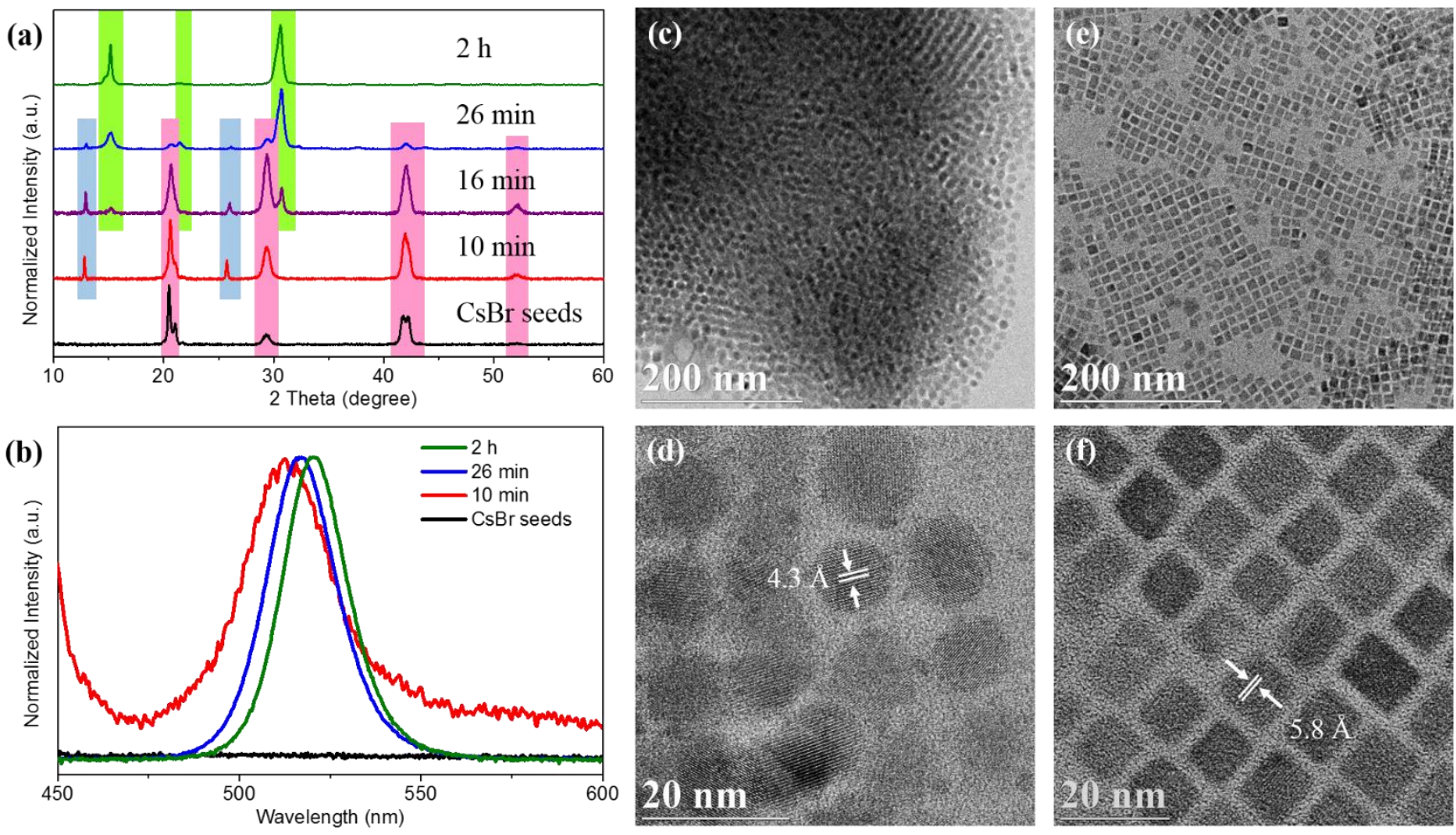

Figure 3. (a) XRD patterns and (b) PL spectra of samples collected at different reaction time in a CsBrseeded growth at $120^{\circ} \mathrm{C}$. In (a), $\mathrm{CsBr}, \mathrm{Cs}_{4} \mathrm{PbBr}_{6}$, and $\mathrm{CsPbBr}_{3}$ patterns are labeled in pink, blue and green, respectively. HRTEM images of $(\mathrm{c}, \mathrm{d}) \mathrm{CsBr}$ seeds and $(\mathrm{e}, \mathrm{f})$ the corresponding seed-grown $\mathrm{CsPbBr}_{3}$ nanocubes. The fringes measured to be $4.3 \AA$ and $5.8 \AA$ correspond to $\left(\begin{array}{lll}1 & 0 & 0\end{array}\right)$ of $\mathrm{CsBr}$ and $\left(\begin{array}{lll}\overline{1} & 0 & \overline{1}\end{array}\right)$ of orthorhombic $\mathrm{CsPbBr}_{3}$, respectively.

These results further demonstrate the generality of our findings with respect to the $\mathrm{Cs}-\mathrm{Pb}-\mathrm{Br}$ hot injection reaction. Bromide complexes with cesium, even in the presence of lead, generating $\mathrm{CsBr}$ seeds (equation (2)). If sufficient bromide is available, the $\mathrm{CsBr}$ seeds react with the additional $\mathrm{Br}^{-}$and existing $\mathrm{Pb}^{2+}$ and are converted first to $\mathrm{Cs}_{4} \mathrm{PbBr}_{6}$ (equation (3)), and then to $\mathrm{CsPbBr}_{3}$ (equation (4)).

$$
\begin{aligned}
& \mathrm{Cs}^{+}+\mathrm{Br}^{-} \rightarrow \mathrm{CsBr} \\
& 4 \mathrm{CsBr}+\mathrm{Pb}^{2+}+2 \mathrm{Br}^{-} \rightarrow \mathrm{Cs}_{4} \mathrm{PbBr}_{6}
\end{aligned}
$$




$$
\mathrm{Cs}_{4} \mathrm{PbBr}_{6}+3 \mathrm{~Pb}^{2+}+6 \mathrm{Br}^{-} \rightarrow 4 \mathrm{CsPbBr}_{3}
$$

Furthermore, the phase conversion reactions can be driven by increasing the concentration of chemically available bromide species in the solution, but not by that of lead. Since raising the concentration of $\mathrm{Pb}$ OA does not force the production of either $\mathrm{Cs}_{4} \mathrm{PbBr}_{6}$ or $\mathrm{CsPbBr}_{3}$, equations (3) \& (4) are independent of or not sensitive to $\mathrm{Pb}-\mathrm{OA}$ concentration.

It has been reported that the crystal phase of products in the system is dependent on the concentrations of $\mathrm{OA}$ and $\mathrm{OAm} .{ }^{23}$ With excess $\mathrm{OA}$ and $\mathrm{OAm}$ in solution, the solvation of $\mathrm{Pb}^{2+}$ and $\mathrm{Br}^{-}$in the liquid phase becomes higher, shifting the equilibrium of the ionic system. In other words, increasing the concentrations of $\mathrm{OAm}$ and $\mathrm{OA}$ drives the reactions $3 \& 4$ in the reverse direction, making $\mathrm{Cs}_{4} \mathrm{PbBr}_{6}$ or $\mathrm{CsBr}_{3}$ the predominant products. It is important to note that the forward reactions are driven by increasing the concentration of bromide, but unaffected by increasing the concentration of lead. As such, we can infer that the equilibrium of bromide between the liquid and solid phases is the key factor that dictates the conversion reactions. This may well explain the facile interconversion between $\mathrm{Cs}_{4} \mathrm{PbBr}_{6}$ and $\mathrm{CsPbBr}_{3}$ achieved by manipulating the ligand concentration in solution, which can be executed even at room temperature, ${ }^{39,40,41}$ since the halides are highly mobile. ${ }^{4}$

Although the direct conversion between these phases within individual nanocrystals has not been observed in situ, the proposed mechanism implies that the bromide-dictated phase conversion reactions may proceed through a surface diffusion route, rather than a pathway of dissolution and subsequent recrystallization. A reaction of pathway of phase conversion was also confirmed by a study in which CsX nanocrystals were transformed to perovskites at room temperature, ${ }^{42}$ and also by the apparent size correlation between $\mathrm{CsX}$ or $\mathrm{Cs}_{4} \mathrm{PbX} 6$ precursors and the $\mathrm{CsPbX}_{3}$ products in other conversion reactions, 
38,42 as also observed here.

In the CsBr-seeded growth study, pure lead-deficient phase nanocrystals were not observed, suggesting that the $\mathrm{Cs}_{4} \mathrm{PbBr}_{6}$ domains are continuously converted into the $\mathrm{CsPbBr}_{3}$ phase during the course of reaction. Interestingly, this proposed mechanism implies that $\mathrm{CsPbr}_{3}$ does not follow a straightforward LaMer mechanism. In fact, the synthesis does not appear to follow a single-species nucleation route, but rather a combination of nucleation, phase conversion, and subsequent growth. This may clarify why, as mentioned previously, many preparation strategies for control over growth employed in other semiconductor materials systems are not applicable to $\mathrm{CsPbBr}_{3}$.

\section{Conclusions}

For the first time, a clear evolution of products in the Cs- $\mathrm{Pb}-\mathrm{Br}$ system was observed. The growth of $\mathrm{Cs}_{\mathrm{PbBr}}$ perovskite nanocrystals starts with the complexation of bromide and cesium, producing $\mathrm{CsBr}$ seeds that subsequently react with bromide and lead to produce $\mathrm{Cs}_{4} \mathrm{PbBr}_{6}$ intermediates, which then convert to $\mathrm{CsPBr}_{3}$. The interconversion of these phases is dictated by the chemical equilibrium of bromide between the liquid and solid phases, and is largely insensitive to the concentration of lead. This study helps elucidate the growth mechanism of $\mathrm{CsPBr}_{3}$ perovskites and provides new insights into inconsistencies with their preparation in comparison with more established colloidal semiconductor nanocrystals, aiding strategies for further chemical and morphological tailorability.

\section{Methods}

\section{Preparation of cesium oleate (Cs-OA).}

$0.1 \mathrm{~g} \mathrm{Cs}_{2} \mathrm{CO}_{3}$ was reacted with $0.3 \mathrm{~mL} \mathrm{OA}$ in $5 \mathrm{~mL} \mathrm{ODE}$ at $120{ }^{\circ} \mathrm{C}$ under vacuum for 1 hour until all of 
chemical was dissolved.

\section{Synthesis of CsPbBr3 under slow kinetics.}

In a typical synthesis, $0.046 \mathrm{~g} \mathrm{PbO}$ was loaded into a $25 \mathrm{~mL}$ flask along with $5 \mathrm{~mL}$ ODE and $0.16 \mathrm{~mL} \mathrm{OA}$, dried at $120{ }^{\circ} \mathrm{C}$ under vacuum for 1.5 hour. Once the chemical was fully dissolved, $0.7 \mathrm{~mL}$ Cs-OA was injected under Ar atmosphere, and the mixture was heated up to $150{ }^{\circ} \mathrm{C} .0 .8 \mathrm{~mL} \mathrm{OAm}$ and $0.6 \mathrm{~mL} \mathrm{OA}$ were added into the solution, and $0.13 \mathrm{~mL} \mathrm{1-bromohexane} \mathrm{was} \mathrm{injected} 2 \mathrm{~min}$ later. Aliquots were taken out at different reaction times and injected into cold toluene solution for temporary storage. To purify the products, samples were centrifuged at $3300 \mathrm{rcf}$ for $20-50 \mathrm{~min}$. The precipitates were redispersed in toluene and underwent another centrifugation cycle. The final precipitates were collected and dispersed in hexane for further characterizations.

\section{Synthesis of CsBr.}

$0.019 \mathrm{~g} \mathrm{PbBr}_{2}$ was loaded into a $25 \mathrm{~mL}$ flask with $2.5 \mathrm{~mL}$ ODE, dried at $120{ }^{\circ} \mathrm{C}$ under vacuum for 1 hour. The flask was then refilled with Ar, and the solution was adjusted to a desired temperature, followed with addition of $0.4 \mathrm{~mL} \mathrm{OAm}$ and $0.1 \mathrm{~mL} \mathrm{OA}$. Once the $\mathrm{PbBr}_{2}$ was fully solubilized, $0.7 \mathrm{~mL}$ of Cs-OA was injected to the mixture and reacted for 2-10 min. The solution would turn cloudy within the first $15 \mathrm{sec}$ to 2 min, depending on the reaction temperature. The product was cooled down in an ice bath, purified using the method mentioned above, and stored in hexane for further characterization. To study the effect of $\mathrm{Pb}$ concentration on the final products, $0.5 \mathrm{~mL} \mathrm{Pb-OA}(0.1822 \mathrm{~g} \mathrm{PbO}, 0.64 \mathrm{~mL} \mathrm{OA}$, and $4.8 \mathrm{~mL}$ ODE) was added to the solution either before or after the injection of Cs-OA.

\section{CsBr-seeded growth of CsPbBr3.}

$\mathrm{CsBr}$ seed solution was prepared at $120^{\circ} \mathrm{C}$ as mentioned above without cooling and purification. To the CsBr crude solution, 0.5 mL Pb-OA (0.1822g PbO, 0.64 mL OA, and 4.8 mL ODE) was added, followed by injection of $0.29 \mathrm{~mL} \mathrm{1-bromohexane.} \mathrm{Aliquots} \mathrm{were} \mathrm{collected} \mathrm{at} \mathrm{different} \mathrm{reaction} \mathrm{time} \mathrm{and} \mathrm{injected}$ 
into cold hexane for temporary storage. Products were centrifuged at $8000 \mathrm{rcf}$ for $10 \mathrm{~min}$ at $20{ }^{\circ} \mathrm{C}$, and the precipitates were dispersed in hexane and underwent another centrifugation cycle at 2500 rcf for 10 min. Precipitates were dispersed in hexane from samples collected in the early stages with no products in the supernatants. At latter stages during the reaction supernatants were also analyzed.

\section{Characterizations}

XRD data was measured on a BRUKER D8-Focus Bragg-Brentano X-ray Powder Diffractometer equipped with $\mathrm{Cu}$ K- $\alpha$ radiation source. PL spectra were collected on an Ocean Optics Flame-S-UV-Vis Spectrometer with an Ocean Optics DH-200-Bal deuterium lamp light source. HRTEM images were taken on a FEI Tecnai G2 F20 ST FE-TEM operated at $200 \mathrm{kV}$ equipped with Gatan CCD camera.

\section{Acknowledgement}

This work is funded in part by the Gordon and Betty Moore Foundation through Grant GBMF6882. M.S. also acknowledges support from the Welch Foundation (A-1886).

\section{Contributions}

J.-R. Wen carried out all the syntheses and characterization works. M. T. Sheldon was responsible for the overall direction of the project. All the other authors participated in preparing the manuscript and contributed to the discussion.

\section{References}

1. Yin, Y. \& Alivisatos, A.P. Colloidal nanocrystal synthesis and the organic-inorganic interface. Nature, 437664 (2004).

2. de Mello Donegá, C., Liljeroth, P. \& Vanmaekelbergh, D. Physicochemical Evaluation of the Hot-Injection Method, a Synthesis Route for Monodisperse Nanocrystals. Small, 1 1152-1162 (2005).

3. Koscher, B. A., Swabeck, J. K., Bronstein, N. D. \& Alivisatos, A. P. Essentially Trap-Free $\mathrm{CsPbBr} 3$ Colloidal Nanocrystals by Postsynthetic Thiocyanate Surface Treatment. J. Am. Chem. Soc., 139 6566-6569 (2017). 
4. Nedelcu, G.et al. Fast Anion-Exchange in Highly Luminescent Nanocrystals of Cesium Lead Halide Perovskites (CsPbX3, X = Cl, Br, I). Nano Lett., 15 5635-5640 (2015).

5. Sarkar, S. et al. Terahertz Spectroscopic Probe of Hot Electron and Hole Transfer from Colloidal CsPbBr3 Perovskite Nanocrystals. Nano Lett., 17 5402-5407 (2017).

6. Becker, M. A. et al. Bright triplet excitons in caesium lead halide perovskites. Nature, 553 189193 (2018).

7. Ye, S. et al. Mechanistic Investigation of Upconversion Photoluminescence in All-Inorganic Perovskite CsPbBrI2 Nanocrystals. J. Phys. Chem. C, 122 3152-3156 (2018).

8. Roman, B. J. \& Sheldon, M. T. The role of mid-gap states in all-inorganic CsPbBr3 nanoparticle one photon up-conversion. ChemComm, 54 6851-6854 (2018).

9. Milstein, T. J., Kroupa, D. M. \& Gamelin, D. R. Picosecond Quantum Cutting Generates Photoluminescence Quantum Yields Over 100\% in Ytterbium-Doped CsPbCl3 Nanocrystals. Nano Lett., $183792-3799$ (2018).

10. Mir, W. J. et al. Postsynthesis Doping of $\mathrm{Mn}$ and $\mathrm{Yb}$ into $\mathrm{CsPbX} 3(\mathrm{X}=\mathrm{Cl}, \mathrm{Br}$, or I) Perovskite Nanocrystals for Downconversion Emission. Chem. Mater., 30 8170-8178 (2018).

11. Akkerman, Q. A., Rainò, G., Kovalenko, M.V. \& Manna, L. Genesis, challenges and opportunities for colloidal lead halide perovskite nanocrystals. Nat. Mater., 17 394-405 (2018).

12. Protesescu, L. et al. Nanocrystals of Cesium Lead Halide Perovskites $(\mathrm{CsPbX} 3, \mathrm{X}=\mathrm{Cl}, \mathrm{Br}$, and I): Novel Optoelectronic Materials Showing Bright Emission with Wide Color Gamut. Nano Lett., 15 3692-3696 (2015).

13. Dong, Y. et al. Precise Control of Quantum Confinement in Cesium Lead Halide Perovskite Quantum Dots via Thermodynamic Equilibrium. Nano Lett., 18 3716-3722 (2018).

14. Zhang, D. D., Eaton, S.W., Yu, Y., Dou, L.T. \& Yang, P.D. Solution-Phase Synthesis of Cesium Lead Halide Perovskite Nanowires. J. Am. Chem. Soc., 137 9230-9233 (2015).

15. Zhang, D.D. et al. Ultrathin Colloidal Cesium Lead Halide Perovskite Nanowires. J. Am. Chem. Soc., 138 13155-13158 (2016).

16. Bekenstein, Y., Koscher, B. A., Eaton, S. W., Yang, P. \& Alivisatos, A.P. Highly Luminescent Colloidal Nanoplates of Perovskite Cesium Lead Halide and Their Oriented Assemblies. J. Am. Chem. Soc., 137 16008-16011 (2015).

17. Shamsi, J. et al. Colloidal Synthesis of Quantum Confined Single Crystal CsPbBr3 Nanosheets with Lateral Size Control up to the Micrometer Range. J. Am. Chem. Soc., 138 7240-7243 (2016). 
18. Cho, J. et al. Influence of ligand shell ordering on dimensional confinement of cesium lead bromide (CsPbBr3) perovskite nanoplatelets. J. Mater. Chem. C, 5 8810-8818 (2017).

19. Parobek, D. et al. Exciton-to-Dopant Energy Transfer in Mn-Doped Cesium Lead Halide Perovskite Nanocrystals. Nano Lett. (2016).

20. Lignos, I. et al. Synthesis of Cesium Lead Halide Perovskite Nanocrystals in a Droplet-Based Microfluidic Platform: Fast Parametric Space Mapping. Nano Lett., 16 1869-1877 (2016).

21. Udayabhaskararao, T., Kazes, M., Houben, L., Lin, H. \& Oron, D. Nucleation, Growth, and Structural Transformations of Perovskite Nanocrystals. Chem. Mater., 29 1302-1308 (2017).

22. Liang, Z. et al. Shape-Controlled Synthesis of All-Inorganic CsPbBr3 Perovskite Nanocrystals with Bright Blue Emission. ACS Appl. Mater. Interfaces, 8 28824-28830 (2016).

23. Almeida, G. et al. Role of Acid-Base Equilibria in the Size, Shape, and Phase Control of Cesium Lead Bromide Nanocrystals. ACS Nano, 12 1704-1711 (2018).

24. Peng, L., Dutta, A., Xie, R., Yang, W. \& Pradhan, N. Dot-Wire-Platelet-Cube: Step Growth and Structural Transformations in CsPbBr3 Perovskite Nanocrystals. ACS Energy Lett., 3 2014-2020 (2018).

25. Liu, Y. et al. Room temperature colloidal synthesis of $\mathrm{CsPbBr} 3$ nanowires with tunable length, width and composition. J. Mater. Chem. C, 6 7797-7802 (2018).

26. Hu, H. C. et al. Interfacial Synthesis of Highly Stable CsPbX3/Oxide Janus Nanoparticles. J. Am. Chem. Soc., 140 406-412 (2018).

27. Zhong, Q. et al. One-Pot Synthesis of Highly Stable CsPbBr3@SiO2 Core-Shell Nanoparticles. ACS Nano, 12 8579-8587 (2018).

28. De Roo, J. et al. Highly Dynamic Ligand Binding and Light Absorption Coefficient of Cesium Lead Bromide Perovskite Nanocrystals. Acs Nano, 10 2071-2081 (2016).

29. Li, Y., Huang, H., Xiong, Y., Kershaw, S.V. \& Rogach, A. L. Revealing the Formation Mechanism of CsPbBr3 Perovskite Nanocrystals Produced via a Slowed-Down MicrowaveAssisted Synthesis. Angew. Chem. Int. Ed., 57 5833-5837 (2018).

30. Sun, C. et al. A new method to discover the reaction mechanism of perovskite nanocrystals. Dalton Trans., 47 16218-16224 (2018).

31. Thanh, N. T. K., Maclean, N. \& Mahiddine, S. Mechanisms of Nucleation and Growth of Nanoparticles in Solution. Chem. Rev., 114 7610-7630 (2014).

32. Imran, M., et al. Benzoyl Halides as Alternative Precursors for the Colloidal Synthesis of Lead- 
Based Halide Perovskite Nanocrystals. J. Am. Chem. Soc., 140 2656-2664 (2018).

33. Dutta, A., Dutta, S. K., Das Adhikari, S. \& Pradhan, N. Tuning the Size of CsPbBr3 Nanocrystals: All at One Constant Temperature. ACS Energy Lett., 3 329-334 (2018).

34. Krieg, F., et al. Colloidal CsPbX3 (X = Cl, Br, I) Nanocrystals 2.0: Zwitterionic Capping Ligands for Improved Durability and Stability. ACS Energy Lett., 3 641-646 (2018).

35. Pan, A., et al. Insight into the Ligand-Mediated Synthesis of Colloidal CsPbBr3 Perovskite Nanocrystals: The Role of Organic Acid, Base, and Cesium Precursors. ACS Nano, 10 79437954 (2016).

36. Lu, C., et al. Cesium Oleate Precursor Preparation for Lead Halide Perovskite Nanocrystal Synthesis: The Influence of Excess Oleic Acid on Achieving Solubility, Conversion, and Reproducibility. Chem. Mater., 31 62-67 (2019).

37. $\mathrm{Wu}$, L., et al. From Nonluminescent Cs4PbX6 (X = Cl, Br, I) Nanocrystals to Highly Luminescent $\mathrm{CsPbX} 3$ Nanocrystals: Water-Triggered Transformation through a CsX-Stripping Mechanism. Nano Lett., 17 5799-5804 (2017).

38. Akkerman, Q. A., et al. Nearly Monodisperse Insulator Cs4PbX6 (X = Cl, Br, I) Nanocrystals, Their Mixed Halide Compositions, and Their Transformation into CsPbX3 Nanocrystals. Nano Lett., 17 1924-1930 (2017).

39. Liu, Z. K., et al. Ligand Mediated Transformation of Cesium Lead Bromide Perovskite Nanocrystals to Lead Depleted Cs4PbBr6 Nanocrystals. J. Am. Chem. Soc., 139 5309-5312 (2017).

40. Palazon, F., et al. Changing the Dimensionality of Cesium Lead Bromide Nanocrystals by Reversible Postsynthesis Transformations with Amines. Chem. Mater., 29 4167-4171 (2017).

41. Udayabhaskararao, T., et al. A Mechanistic Study of Phase Transformation in Perovskite Nanocrystals Driven by Ligand Passivation. Chem. Mater., 30 84-93 (2018).

42. Shamsi, J., et al. Colloidal CsX $(\mathrm{X}=\mathrm{Cl}, \mathrm{Br}, \mathrm{I})$ Nanocrystals and Their Transformation to CsPbX3 Nanocrystals by Cation Exchange. Chem. Mater., 30 79-83 (2018). 
Chemical Availability of Bromide Dictates CsPbBr3 Nanocr... (1.08 MiB) view on ChemRxiv • download file 
Supporting Information for:

\section{Chemical Availability of Bromide Dictates $\mathrm{CsPbBr}_{3}$ Nanocrystal Growth}

Je-Ruei Wen, ${ }^{\dagger}$ Benjamin J. Roman,$^{\dagger}$ Freddy A. Rodriguez Ortiz, ${ }^{\dagger}$ Noel Mireles Villegas, ${ }^{\dagger}$ Nicholas Porcellino, ${ }^{\dagger}$ Matthew T. Sheldon* ${ }^{\dagger}$

$\dagger$ Department of Chemistry and Department of Material Science and Engineering, Texas A\&M University, College Station, Texas 77843, United States.

\section{Materials}

$\mathrm{Cs}_{2} \mathrm{CO}_{3}(99.9 \%), \mathrm{PbO}(99 \%)$, oleic acid (OA, 90\%), oleylamine (OAm, 70\%), 1-octadecene (ODE, 90\%), toluene (99.8\%) and hexane (95\%) were received from Sigma-Aldrich. 1-bromohexane (99\%) and $\mathrm{PbBr}_{2}(98+\%)$ were purchased from Alfa Aesar. OA and OAm were dried with molecular sieves under Ar environment before use. Other chemicals were used as received.

\section{Control experiment.}

Cs-OA and $\mathrm{Pb}-\mathrm{OA}$ were prepared as described above and kept at $150{ }^{\circ} \mathrm{C}$ in Ar environment. To the Pb-OA solution, $0.8 \mathrm{~mL}$ OAm, $0.6 \mathrm{~mL} \mathrm{OA}$, and $0.13 \mathrm{~mL}$ 1-bromohexane were added, and the mixture was aged at $150{ }^{\circ} \mathrm{C}$ for $30 \mathrm{~min} .0 .7 \mathrm{~mL}$ of Cs-OA was then injected into the mixture, which would turn fluorescent green right away. Aliquots were taken out and injected into cold toluene solution for temporary storage. To purify the products, samples were centrifuged at $3300 \mathrm{rcf}$ for $20-50 \mathrm{~min}$. The precipitates were redispersed in toluene and underwent another centrifugation cycle. The final precipitates were collected and dispersed in hexane for further characterizations. 


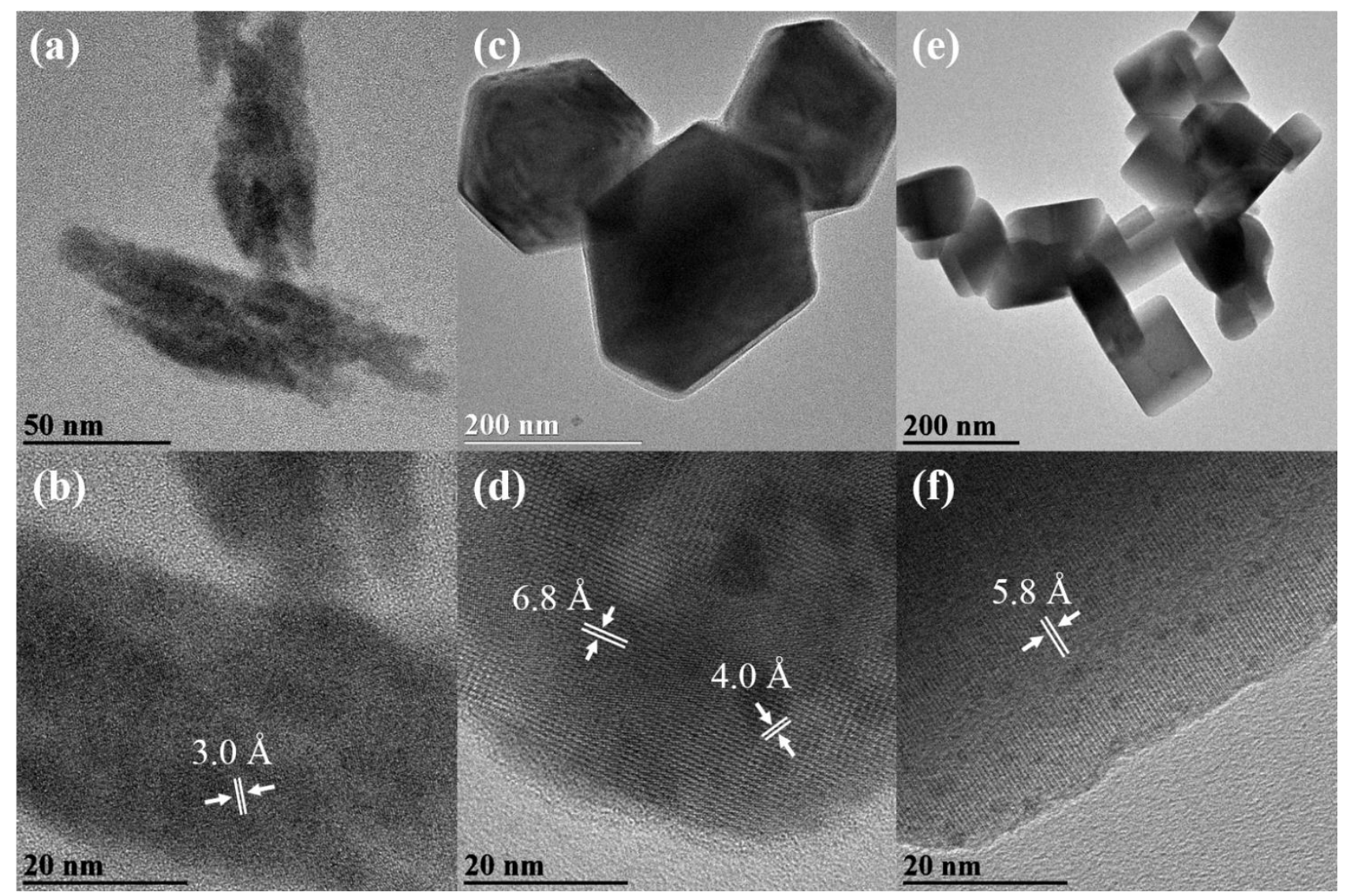

Figure S1. HRTEM images of samples collected at (a,b) 2, (c,d) 8.5, and (e,f) 20 min. 
Table S1. Reaction parameters. The units are in mmol.

\begin{tabular}{|c|c|c|c|c|c|}
\hline sample & $\mathbf{C s}$ & $\mathbf{P b}$ & 1-bromohexane & OA & OAm \\
\hline Typical & 0.11 & 0.202 & 0.91 & 2.17 & 1.70 \\
\hline Modified & 0.11 & 0.203 & 1.40 & 2.17 & 0.21 \\
\hline
\end{tabular}
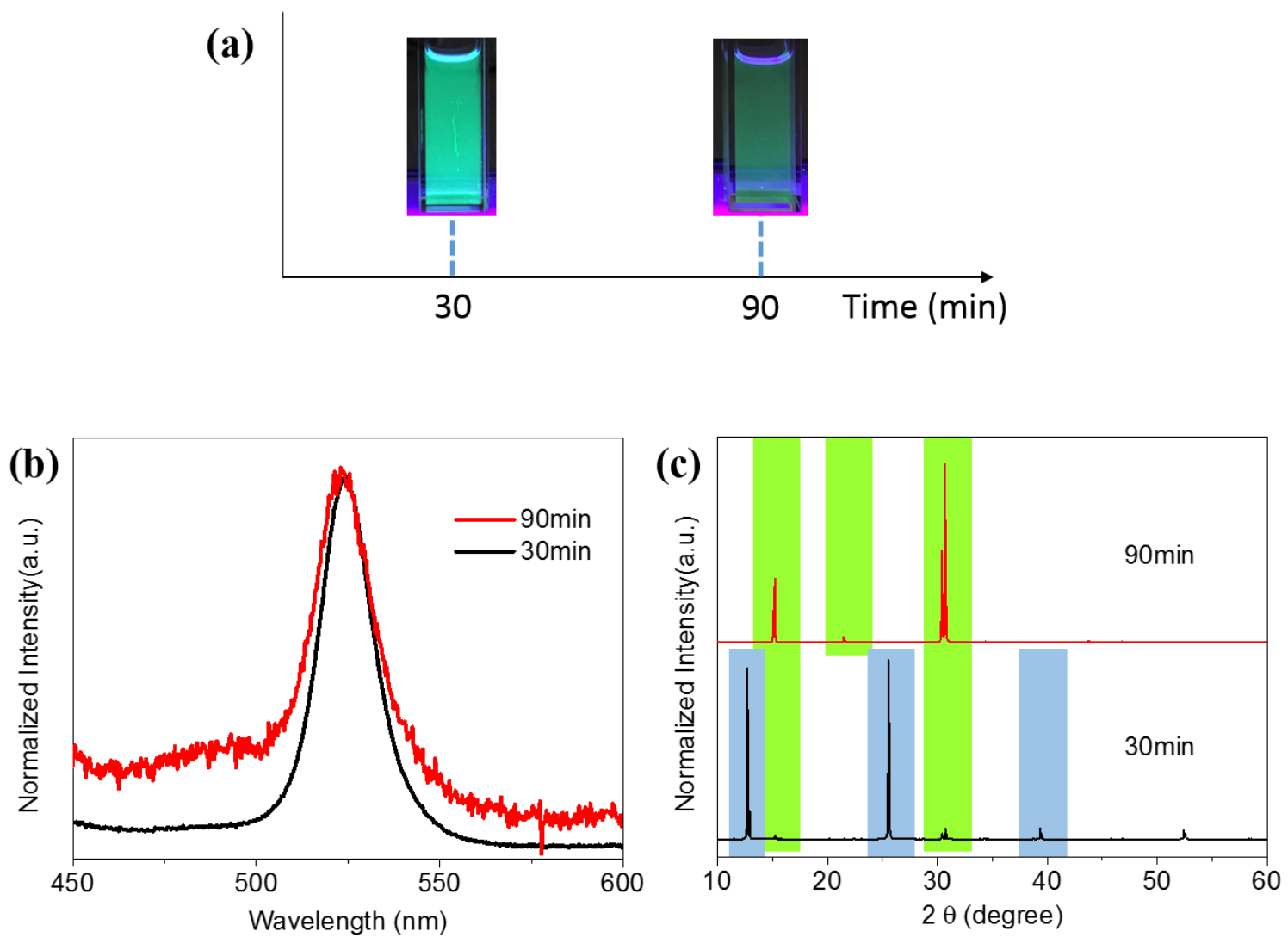

Figure S2. (a) Photos, (b) PL spectra, and (c) XRD patterns of the sample with modified reaction parameters collected at 30 and $90 \mathrm{~min}$. The photos were taken under UV light illumination. In (c), $\mathrm{Cs}_{4} \mathrm{PbBr}_{6}$ and $\mathrm{CsPbBr}_{3}$ signals are labeled in blue and green, respectively. 


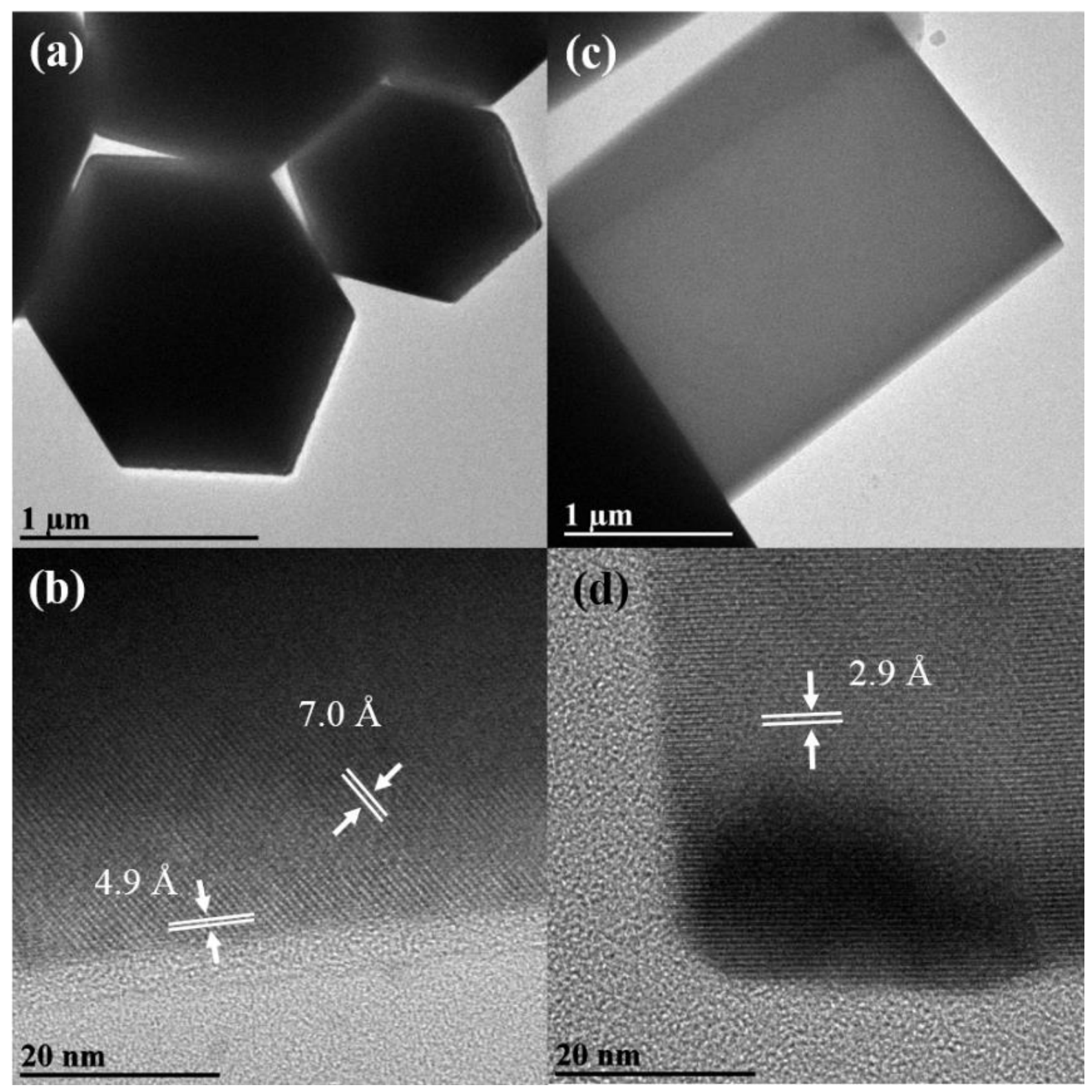

Figure S3. HRTEM images of the sample with modified reaction parameters collected at (a,b) 30 and (c,d) $90 \mathrm{~min}$. The lattice fringes of 7.0 and $4.9 \AA$ found in (b) can be assigned to $\left(\begin{array}{lll}2 & 0 & 2\end{array}\right)$ and $\left(\begin{array}{lll}0 & 1 & 2\end{array}\right)$ of hexagonal $\mathrm{Cs}_{4} \mathrm{PbBr}_{6}$, respectively, while the fringe measured to be 2.9 $\AA$ in $(\mathrm{d})$ corresponds to $(\overline{2} 0 \overline{2})$ of orthorhombic $\mathrm{CsPbBr}_{3}$. 


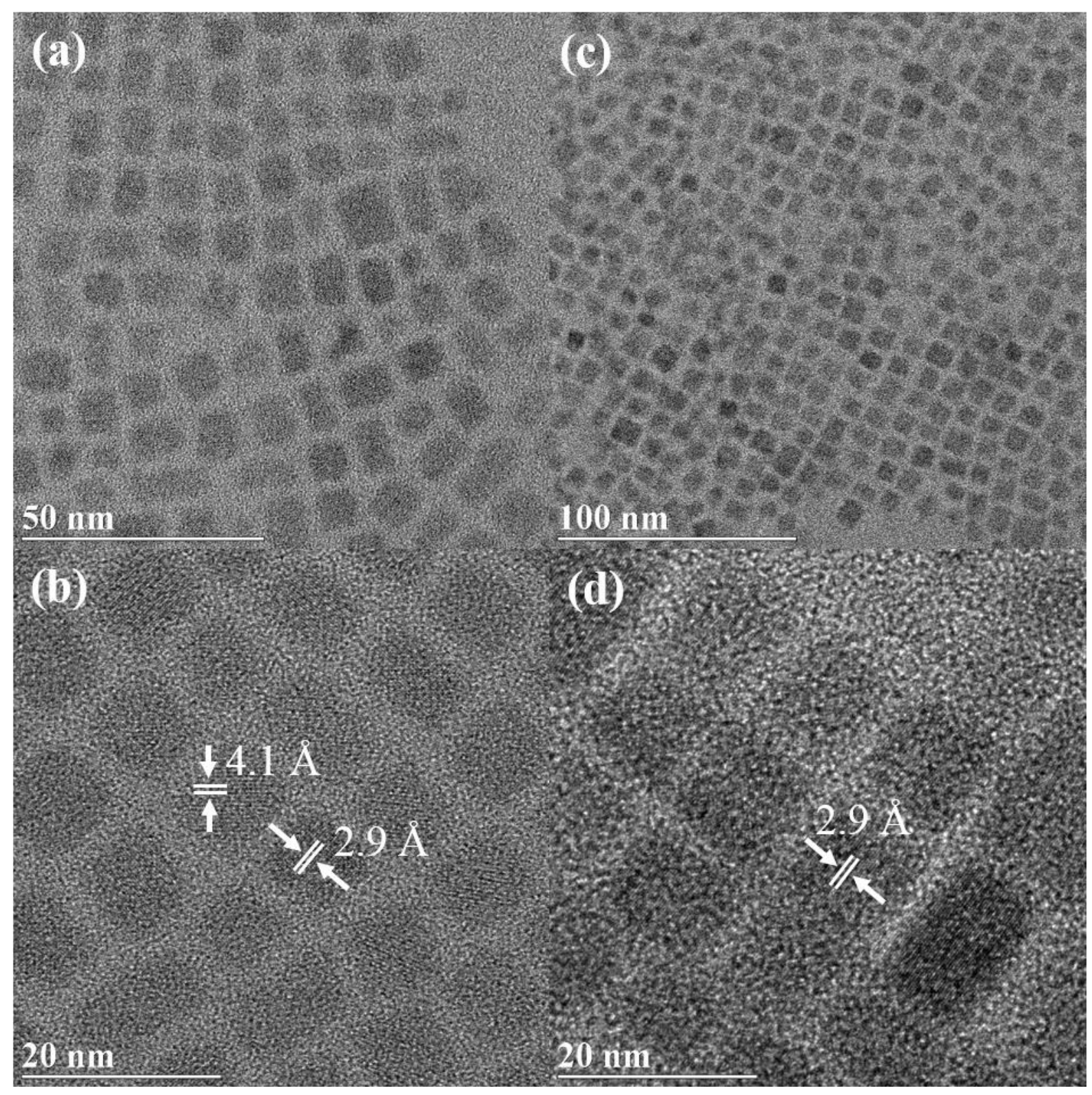

Figure S4. HRTEM images of the control sample collected at (a,b) $30 \mathrm{sec}$ and (c,d) $30 \mathrm{~min}$. The lattice fringes of 4.1 and $2.9 \AA$ are assigned to $(\overline{2} 00)$ and $(\overline{2} 0 \overline{2})$ of orthorhombic $\mathrm{CsPbBr}_{3}$, respectively. 




Figure S5. XRD patterns of CsBr nanocrystals prepared at 80 and $150{ }^{\circ} \mathrm{C}$.

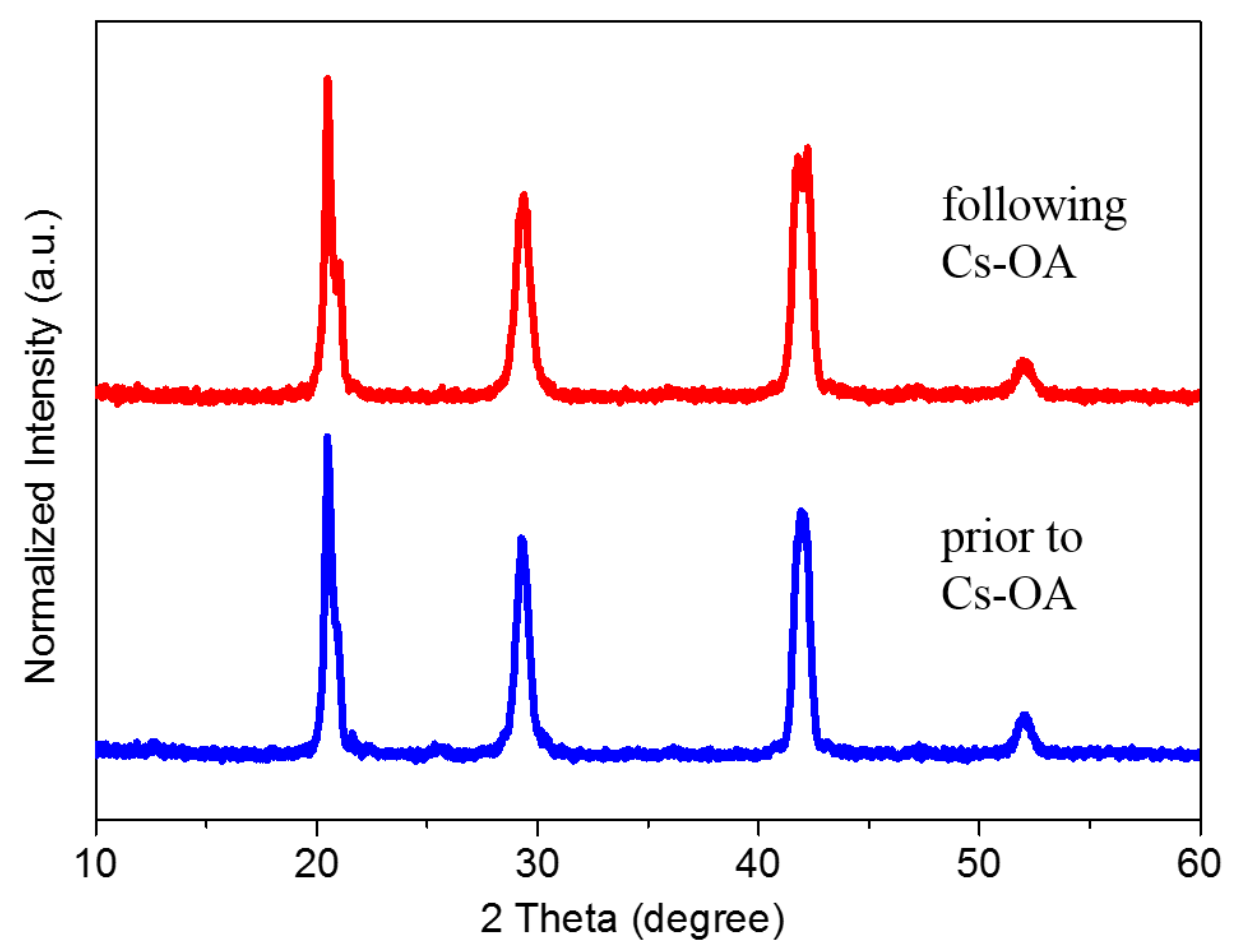

Figure S6. XRD patterns of $\mathrm{CsBr}$ nanocrystals prepared at $120{ }^{\circ} \mathrm{C}$ with addition of extra $\mathrm{Pb}-\mathrm{OA}$ prior to or following the injection of $\mathrm{Cs}-\mathrm{OA}$. The final molar ratio of $\mathrm{Cs}: \mathrm{Pb}: \mathrm{Br}$ in the mixture is $1: 1.2: 1$. 
SI --Chemical Availability of Bromide Dictates $\mathrm{CsPbBr} 3 \mathrm{Na} \ldots$ (1.04 MiB) view on ChemRxiv • download file 